\title{
Ranking and Sparsifying a Connection Graph
}

\author{
Fan Chung, Wenbo Zhao, and Mark Kempton
}

\begin{abstract}
Many problems arising in dealing with high-dimensional data sets involve connection graphs in which each edge is associated with both an edge weight and a $d$-dimensional linear transformation. We consider vectorized versions of PageRank and effective resistance that can be used as basic tools for organizing and analyzing complex data sets. For example, generalized PageRank and effective resistance can be utilized to derive and modify diffusion distances for vector diffusion maps in data and image processing. Furthermore, the edge-ranking of the connection graphs determined by vectorized PageRank and effective resistance are an essential part of sparsification algorithms that simplify and preserve the global structure of connection graphs. In addition, we examine consistencies in a connection graph, particularly in the applications of recovering low-dimensional data sets and the reduction of noise. In these applications, we analyze the effect of deleting edges with high edge rank.
\end{abstract}

\section{Introduction}

In this paper, we consider a generalization of graphs, called connection graphs, in which each edge of the graph is associated with a weight and also a "rotation" (which is a linear orthogonal transformation acting on a $d$-dimensional vector space for some positive integer $d$ ). The adjacency matrix and the discrete Laplace operator are linear operators acting on the space of vector-valued

(C) Taylor \& Francis Group, LLC

ISSN: I542-795I print 
functions (instead of the usual real-valued functions) and therefore can be represented by matrices of size $d n \times d n$, where $n$ is the number of vertices in the graph.

Connection graphs arise in numerous applications, in particular for data and image processing involving high-dimensional data sets. To quantify the affinities between two data points, it is often not enough to use only a scalar edge weight. For example, if the high-dimensional data set can be represented or approximated by a low-dimensional manifold, the patterns associated with nearby data points are likely to related by certain rotations [Singer and $\mathrm{Wu} 12$ ]. There are many recent developments of related research in electron cryomicroscopy [Hadani and Singer 11, Singer et al. 11], angular synchronization of eigenvectors [Cucuringu et al. 12, Singer 11], and vector diffusion maps [Singer and Wu 12]. In many areas of machine learning, high-dimensional data points in general can be treated by various methods, such as principal component analysis [Jolliffe 02], to reduce vectors into some low-dimensional space and then use the connection graph with rotations on edges to provide additional information for proximity. In computer vision, there has been a great deal of recent work dealing with trillions of photos that are now available on the Web [Agarwal et al. 09]. Feature-matching techniques [Lowe 99] can be used to derive vectors associated with the images. Then information networks of photos can be built that are precisely connection graphs with rotations corresponding to the angles and positions of the cameras in use. The use of connection graphs can be further traced to earlier work in graph gauge theory for computing the vibrational spectra of molecules and examining the spins associated with vibrations [Chung and Sternberg 92].

Many information networks arising from massive data sets exhibit the smallworld phenomenon. Consequently, the usual graph distance is no longer very useful. It is crucial to have an appropriate metric for expressing the proximity between two vertices. Various notions of diffusion distances have been defined [Singer and $\mathrm{Wu} 12$ ] and used for manifold learning and dimension reduction. Here we consider two basic notions, connection PageRank and connection resistance (which are generalizations of the usual PageRank and effective resistance). Both connection PageRank and connection resistance can then be used to measure relationships between vertices in a connection graph. To illustrate the use of both metrics, we derive edge-ranking using connection PageRank and connection resistance. In the applications to electron cryomicroscopy, edge-ranking can help eliminate the superfluous or erroneous edges that appear because of various types of "noise." We will use here connection PageRank and connection resistance as tools for the basis of algorithms that can be used to construct a sparsifier that has fewer edges but preserves the global structure of the connection network. 
The notion of PageRank was first introduced in [Brin and Page 98] for Google's Web search algorithms. Although PageRank was originally designed for the Web graph, the concepts work well for any graph for quantifying the relationships between pairs of vertices (or pairs of subsets) in any given graph. There are very efficient and robust algorithms for computing and approximating PageRank [Andersen et al. 06, Berkhin 06, Jeh and Widom 03, Borgs et al. 12]. In this paper, we further generalize PageRank for connection graphs and give efficient and sharp approximation algorithms for computing the connection PageRank, similar to the algorithm presented in [Borgs et al. 12].

Effective resistance plays a major role in electrical network theory and can be traced back to the classical work [Kirchhoff 47]. Here we consider a generalized version of effective resistance for connection graphs. To illustrate the use of connection resistance, we examine a basic problem on graph sparsification. Graph sparsification was introduced in [Benczúr and Karger 96, Karger 99, Karger 94, Karger 00] for approximately solving various network design problems. The heart of graph-sparsification algorithms is a sampling technique for randomly selecting edges. The goal is to approximate a given graph $G$ on $n$ vertices by a sparse graph $\tilde{G}$, called a sparsifier, with fewer edges on the same set of vertices such that every cut in the sparsifier $\tilde{G}$ has size within a factor $1 \pm \epsilon$ of the size of the corresponding cut in $G$ for some constant $\epsilon$. In [Spielman and Teng 04], the authors constructed a spectral sparsifier with $\mathcal{O}\left(n \log ^{c} n\right)$ edges for some large constant $c$. In [Spielman and Srivastava 08], the authors gave a different sampling scheme using effective resistances to construct an improved spectral sparsifier with only $\mathcal{O}(n \log n)$ edges. In this paper, we will construct a connection sparsifier using the weighted connection resistance. Our algorithm is similar to that found in [Spielman and Srivastava 08].

In recent work [Bandeira et al. 12], the authors study the $\mathrm{O}(d)$ synchronization problem in which each vertex of a connection graph is assigned a rotation in the orthogonal group $\mathrm{O}(d)$. Our work differs from theirs in that here, we examine the problem of assigning a vector in $\mathbf{R}^{d}$ to each vertex, rather than an orthogonal matrix in $\mathrm{O}(d)$ (see the remark following the proof of Theorem 2.2). In other words, our connection Laplacian is an operator acting on the space of vectorvalued functions. However, their work is closely related to our work in this paper. In particular, they define the connection Laplacian and use its spectrum to give a measure of how close a connection graph is to being consistent.

\section{I.I. A Summary of Our Results}

Our results can be summarized as follows: We review definitions for the connection graph and the connection Laplacian in Section 2. The connection 
Laplacian is also studied in [Singer and Wu 12, Bandeira et al. 12]. In particular, we discuss the notion of "consistency" in a connection graph (which is considered to be the ideal situation for various applications). We give a characterization for a consistent connection graph using the eigenvalues of the connection Laplacian.

We introduce the connection PageRank in Section 3. We follow the method of [Borgs et al. 12] to develop a sublinear-time algorithm for computing an approximate connection PageRank vector. We define the connection resistance in Section 4 and then examine various properties of the connection resistance. We use the connection resistance to give an edge-ranking algorithm and a sparsification algorithm for connection graphs in Section 5 .

In Section 6, we propose a method for reducing noise in data by deleting, with high probability, edges having large edge rank. Using probabilistic and spectral techniques as in [Chung and Radcliffe 11], we prove that for a connection graph, the eigenvalue related to consistency can be substantially reduced by deleting edges with high rank. Consequently, the resulting graph is an improved approximation for recovering a consistent connection graph.

\section{Preliminaries}

For positive integers $m, n, d$, we consider a family of matrices, which we denote by $\mathcal{F}(m, n, d ; \mathbf{R})$, consisting of all $m d \times n d$ matrices with real-valued entries. A matrix in $\mathcal{F}(m, n, d ; \mathbf{R})$ can also be viewed as an $m \times n$ matrix whose entries are represented by $d \times d$ blocks. A rotation is a matrix that is used to perform a rotation in Euclidean space. Namely, a rotation $O$ is a square matrix, with real entries, satisfying $O^{T}=O^{-1}$ and $\operatorname{det}(O)=1$. The set of $d \times d$ rotation matrices forms the special orthogonal group $\mathrm{SO}(d)$. It is easy to check that all eigenvalues of a rotation $O$ are of norm 1. Furthermore, a rotation $O \in \mathrm{SO}(d)$ with $d$ odd has an eigenvalue 1 (see [Golub and Van Loan 96]).

\section{I. The Connection Laplacian}

Suppose $G=(V, E, w)$ is an undirected graph with vertex set $V$, edge set $E$, and edge weights $w_{u v}=w_{v u}>0$ for edges $(u, v)$ in $E$. Suppose each oriented edge $(u, v)$ is associated with a rotation matrix $O_{u v} \in \mathrm{SO}(d)$ satisfying $O_{u v} O_{v u}=$ $I_{d \times d}$. Let $O$ denote the set of rotations associated with all oriented edges in $G$. The connection graph, denoted by $\mathbb{G}=(V, E, O, w)$, has $G$ as the underlying 
graph. The connection matrix $\mathbb{A}$ of $\mathbb{G}$ is defined by

$$
\mathbb{A}(u, v)= \begin{cases}w_{u v} O_{u v} & \text { if }(u, v) \in E, \\ 0_{d \times d} & \text { if }(u, v) \notin E,\end{cases}
$$

where $0_{d \times d}$ is the zero matrix of size $d \times d$. In other words, for $|V|=n$, we view $\mathbb{A} \in \mathcal{F}(n, n, d ; \mathbf{R})$ as a block matrix each block of which is either a $d \times d$ rotation matrix $O_{u v}$ multiplied by a scalar weight $w_{u v}$ or a $d \times d$ zero matrix. The matrix $\mathbb{A}$ is symmetric, since $O_{u v}^{T}=O_{v u}$ and $w_{u v}=w_{v u}$. The diagonal matrix $\mathbb{D} \in \mathcal{F}(n, n, d ; \mathbf{R})$ is defined by the diagonal blocks $\mathbb{D}(u, u)=d_{u} I_{d \times d}$ for $u \in V$. Here $d_{u}$ is the weighted degree of $u$ in $G$, i.e., $d_{u}=\sum_{(u, v) \in E} w_{u v}$.

The connection Laplacian $\mathbb{L} \in \mathcal{F}(n, n, d ; \mathbf{R})$ of a graph $\mathbb{G}$ is the block matrix $\mathbb{L}=\mathbb{D}-\mathbb{A}$. Recall that for a given orientation of edges of the underlying graph $G$ on $n$ vertices and $m$ edges, the combinatorial Laplacian $L$ can be written as $L=B^{T} W B$, where $W$ is an $m \times m$ diagonal matrix with $W_{e, e}=w_{e}$, and $B$ is the edge--vertex incidence matrix of size $m \times n$ such that

$$
B(e, v)= \begin{cases}1 & \text { if } v \text { is } e \text { 's head } \\ -1 & \text { if } v \text { is } e \text { 's tail } \\ 0 & \text { otherwise. }\end{cases}
$$

A useful observation for the connection Laplacian is the fact that it can be written in a similar form. Let $\mathbb{B} \in \mathcal{F}(m, n, d ; \mathbf{R})$ be the block matrix given by

$$
\mathbb{B}(e, v)= \begin{cases}O_{u v} & v \text { is } e \text { 's head, } \\ -I_{d \times d} & v \text { is } e \text { 's tail, } \\ 0_{d \times d} & \text { otherwise. }\end{cases}
$$

Also, let the block matrix $\mathbb{W} \in \mathcal{F}(m, m, d ; \mathbf{R})$ denote a diagonal block matrix given by $\mathbb{W}(e, e)=w_{e} I_{d \times d}$. We remark that given an orientation of the edges, the connection Laplacian can alternatively be defined as

$$
\mathbb{L}=\mathbb{B}^{T} \mathbb{W} \mathbb{B} .
$$

This can be verified by direct computation.

We have the following useful lemma regarding the Dirichlet sum of the connection Laplacian as an operator on the space of vector-valued functions on the vertex set of a connection graph.

Lemma 2.I. For every function $f: V \rightarrow \mathbf{R}^{d}$, we have

$$
f \llbracket f^{T}=\sum_{(u, v) \in E} w_{u v}\left\|f(u) O_{u v}-f(v)\right\|_{2}^{2},
$$


where $f(v)$ here is regarded as a row vector of dimension d. Furthermore, an eigenpair $\left(\lambda_{i}, \phi_{i}\right)$ has $\lambda_{i}=0$ if and only if $\phi_{i}(u) O_{u v}=\phi_{i}(v)$ for all $(u, v) \in E$.

Proof.

For (2.1), observe that for a fixed edge $e=(u, v)$, we have

$$
f \mathbb{B}^{T}(e)=f(u) O_{u v}-f(v) .
$$

Thus,

$$
\begin{aligned}
f \mathbb{L} f^{T} & =\left(f \mathbb{B}^{T}\right) \mathbb{W}\left(\mathbb{B} f^{T}\right)=\left(f \mathbb{B}^{T}\right) \mathbb{W}\left(f \mathbb{B}^{T}\right)^{T} \\
& =\sum_{(u, v) \in E} w(u, v)\left\|f(u) O_{u v}-f(v)\right\|_{2}^{2} .
\end{aligned}
$$

Also, $\mathbb{L}$ is symmetric and therefore has real eigenfunctions and real eigenvalues. The spectral decomposition of $\mathbb{L}$ is given by

$$
\mathbb{L}_{\mathbb{G}}(u, v)=\sum_{i=1}^{n d} \lambda_{i} \phi_{i}(u)^{T} \phi_{i}(v) .
$$

By (2.1)), $\lambda_{1} \geq 0$ and $\lambda_{i}=0$ if and only if $\phi_{i}(u) O_{u v}=\phi_{i}(v)$ for all $\{u, v\} \in E$, and the lemma follows.

\subsection{The Consistency of a Connection Graph}

For a connection graph $\mathbb{G}=(V, E, O, w)$, we say that $\mathbb{G}$ is consistent if for every cycle $c=\left(v_{k}, v_{1}, v_{2}, \ldots, v_{k}\right)$, the product of rotations along the cycle is the identity matrix, i.e.,

$$
O_{v_{k} v_{1}} \prod_{i=1}^{k-1} O_{v_{i} v_{i+1}}=I_{d \times d} .
$$

In other words, for two vertices $u$ and $v$, the products of rotations along different paths from $u$ to $v$ are the same. In the following theorem, we give a characterization of a consistent connection graph using the eigenvalues of the connection Laplacian.

Theorem 2.2. Let $\mathbb{G}$ be a connected connection graph on $n$ vertices having connection Laplacian $\mathbb{L}$ of dimension $n d$, and let $L$ be the Laplacian of the underlying graph $G$. The following statements are equivalent:

(i) $\mathbb{G}$ is consistent.

(ii) The connection Laplacian $\mathbb{L}$ of $\mathbb{G}$ has d eigenvalues of value 0 . 
(iii) The eigenvalues of $\mathbb{L}$ are the $n$ eigenvalues of $L$, each of multiplicity $d$.

(iv) For each vertex $u$ in $G$, we can find $O_{u} \in \mathrm{SO} d$ such that for every edge $(u, v)$ with rotation $O_{u v}$, we have $O_{u v}=O_{u}^{-1} O_{v}$.

Proof. (i) $\Longrightarrow$ (ii). For a fixed vertex $u \in V$ and an arbitrary $d$-dimensional vector $\widehat{x}$, we can define a function $\widehat{f}: V \rightarrow \mathbf{R}^{d}$ by defining $\widehat{f}(u)=\widehat{x}$ initially. Then we assign $\widehat{f}(v)=\widehat{f}(u) O_{u v}$ for all the neighbors $v$ of $u$. Since $G$ is connected and $\mathbb{G}$ is consistent, we can continue the assigning process to all neighboring vertices without any conflict until all vertices are assigned. The resulting function $\widehat{f}: V \rightarrow \mathbf{R}^{d}$ satisfies

$$
\widehat{f} \llbracket \widehat{f}^{T}=\sum_{(u, v) \in E} w_{u v}\left\|\widehat{f}(u) O_{u v}-\widehat{f}(v)\right\|_{2}^{2}=0 .
$$

Therefore, 0 is an eigenvalue of $\mathbb{L}$ with eigenfunction $\widehat{f}$. There are $d$ orthogonal choices for the initial choice of $\widehat{x}=\widehat{f}(u)$. Therefore, we obtain $d$ orthogonal eigenfunctions $\widehat{f_{1}}, \ldots, \widehat{f_{d}}$ corresponding to the eigenvalue 0 .

(ii) $\Longrightarrow$ (iii). Let us consider the underlying graph $G$. Let $f_{i}: V \rightarrow \mathbf{R}$ denote the eigenfunctions of $L$ corresponding to the eigenvalue $\lambda_{i}$ for $i \in[n]$. Let $\widehat{f}_{k}$, for $k \in[d]$, be orthogonal eigenfunctions of $\mathbb{L}$ for the eigenvalue 0. By Lemma 2.1, each $\widehat{f}_{k}$ satisfies $\widehat{f}_{k}(u) O_{u v}=\widehat{f}_{k}(v)$. Our proof of this part follows directly from the following claim.

Claim 2.3. Functions $f_{i} \otimes \widehat{f}_{k}: V \rightarrow \mathbf{R}^{d}$ for $i \in[n], k \in[d]$ are the orthogonal eigenfunctions of $\mathbb{L}$ corresponding to eigenvalue $\lambda_{i}$, where $f_{i} \otimes \widehat{f}_{k}(v)=f_{i}(v) \widehat{f}_{k}(v)$.

Proof. First, we need to verify that the functions $f_{i} \otimes \widehat{f}_{k}$ are eigenfunctions of $\mathbb{L}$. We note that

$$
\begin{aligned}
{\left[f_{i} \otimes \widehat{f}_{k} \mathbb{L}\right](u) } & =d(u) f_{i} \otimes \widehat{f}_{k}(u)-\sum_{v \sim u} w_{v u} f_{i} \otimes \widehat{f}_{k}(v) O_{v u} \\
& =d(u) f_{i}(u) \widehat{f}_{k}(u)-\sum_{v \sim u} w_{v u} f_{i}(v) \widehat{f}_{k}(v) O_{v u} \\
& =d(u) f_{i}(u) \widehat{f}_{k}(u)-\sum_{v \sim u} w_{v u} f_{i}(v) \widehat{f}_{k}(u) \\
& =\left(d(u) f_{i}(u)-\sum_{v \sim u} w_{v u} f_{i}(v)\right) \widehat{f}_{k}(u) .
\end{aligned}
$$


Since $f_{i}$ is an eigenfunction of $L$ corresponding to the eigenvalue $\lambda_{i}$, we have $f_{i} L=\lambda_{i} f_{i}$, i.e.,

$$
\left(d(u) f_{i}(u)-\sum_{v \sim u} w_{v u} f_{i}(v)\right)=\lambda_{i} f_{i}(u) .
$$

Thus,

$$
\left[f_{i} \otimes \widehat{f}_{k} \mathbb{}\right](u)=\lambda_{i} f_{i}(u) \widehat{f}_{k}(u)=\lambda_{i} f_{i} \otimes \widehat{f}_{k}(u),
$$

and $f_{i} \otimes \widehat{f}_{k}, 1 \leq i \leq n, 1 \leq k \leq d$, are the eigenfunctions of $\mathbb{L}$ with eigenvalue $\lambda_{i}$.

To prove the orthogonality of the $f_{i} \otimes \widehat{f}_{k}$, we note that if $k \neq l$, then

$$
\begin{aligned}
\left\langle f_{i} \otimes \widehat{f}_{k}, f_{j} \otimes \widehat{f}_{l}\right\rangle & =\sum_{v}\left\langle f_{i} \otimes \widehat{f}_{k}(v), f_{j} \otimes \widehat{f}_{l}(v)\right\rangle=\sum_{v} f_{i}(v) f_{j}(v)\left\langle\widehat{f}_{k}(v), \widehat{f}_{l}(v)\right\rangle \\
& =0,
\end{aligned}
$$

since $\left\langle\widehat{f_{k}}(v), \widehat{f_{l}}(v)\right\rangle=0$ for $k \neq l$. For the case $k=l$ but $i \neq j$, we have

$$
\left\langle f_{i} \otimes \widehat{f}_{k}, f_{j} \otimes \widehat{f}_{l}\right\rangle=\sum_{v} f_{i}(v) f_{j}(v)\left\langle\widehat{f}_{k}(v), \widehat{f}_{k}(v)\right\rangle=\sum_{v} f_{i}(v) f_{j}(v)=0,
$$

because of $\left\langle f_{i}, f_{j}\right\rangle=0$ for $i \neq j$. The claim is proved.

(iii) $\Longrightarrow$ (iv). Since 0 is an eigenvalue of $L$, we can let $\widehat{f}_{1}, \ldots, \widehat{f}_{d}$ be $d$ orthogonal eigenfunctions of $\mathbb{L}$ corresponding to the eigenvalue 0. By Lemma 2.1, $\widehat{f}_{k}(u) O_{u v}=\widehat{f}_{k}(v)$ for all $k \in[d], u v \in E$. For two adjacent vertices $u$ and $v$, we have, for $i, j=1, \ldots, d$,

$$
\left\langle\widehat{f}_{i}(u), \widehat{f}_{j}(u)\right\rangle=\left\langle\widehat{f}_{i}(u) O_{u v}, \widehat{f}_{j}(u) O_{u v}\right\rangle=\left\langle\widehat{f}_{i}(v), \widehat{f}_{j}(v)\right\rangle .
$$

Therefore, $\widehat{f}_{1}(v), \ldots, \widehat{f}_{d}(v)$ must form an orthogonal basis of $\mathbf{R}^{d}$ for all $v \in V$.

So for $v \in V$, define $O_{v}$ to be the matrix with rows $\widehat{f}_{1}(v), \ldots, \widehat{f}_{d}(v)$, and if necessary, normalize and adjust the signs of these vectors to guarantee that $O_{v} \in \mathrm{SO}(d)$. Then $O_{v}$ is an orthogonal matrix for each $d$, and for an edge $u v \in E$, $O_{u} O_{u v}=O_{v}$, which implies $O_{u v}=O_{u}^{-1} O_{v}$.

(iv) $\Longrightarrow$ (i). Let $C=\left(v_{1}, v_{2}, \ldots, v_{k}, v_{1}\right)$ be a cycle in $G$. Then

$$
O_{v_{k} v_{1}} \prod_{i=1}^{k-1} O_{v_{i} v_{i+1}}=O_{v_{k}}^{-1} O_{v_{1}} \prod_{i=1}^{k-1} O_{v_{i}}^{-1} O_{v_{i+1}}=I_{d \times d} .
$$

Therefore, $\mathbb{G}$ is consistent. This completes the proof of the theorem.

We note that item (iv) in the previous result is related to the $\mathrm{O}(d)$ synchronization problem studied in [Bandeira et al. 12]. This problem consists in finding a function $O: V(G) \rightarrow \mathrm{O}(d)$ such that given the offsets $O_{u v}$ in the edges, the 
function satisfies $O_{u v}=O_{u}^{-1} O_{v}$. The previous theorem shows that this has an exact solution if $\mathbb{G}$ is consistent. Particularly, [Bandeira et al. 12] investigates how well a solution can be approximated when the connection graph is not consistent. The formulation in that work gives a measure of how close a connection graph is to being consistent by looking at the operator on the space of functions $O: V(G) \rightarrow \mathrm{O}(d)$ given by $\sum_{u \sim v} w_{u v}\left\|O_{u} O_{u v}-O_{v}\right\|_{2}^{2}$. In order to investigate this, the authors also considered the operator on the space of vector-valued functions $f: V(G) \rightarrow \mathbb{R}^{d}$ given by $\sum_{u \sim v} w_{u v}\left\|f_{u} O_{u v}-f_{v}\right\|_{2}^{2}$, which is what we are using to investigate the connection Laplacian.

\subsection{Random Walks on a Connection Graph}

Consider the underlying graph $G$ of a connection graph $\mathbb{G}=(V, E, O, w)$. A random walk on $G$ is defined by the transition probability matrix $P$, where $P_{u v}=w_{u v} / d_{u}$ denotes the probability of moving to a neighbor $v$ at a vertex $u$. We can write $P=D^{-1} A$, where $A$ is the weighted adjacency matrix of $G$, and $D$ is the diagonal matrix of weighted degree.

In a similar way, we can define a random walk on the connection graph $\mathbb{G}$ by setting the transition probability matrix $\mathbb{P}=\mathbb{D}^{-1} \mathbb{A}$. While $P$ acts on the space of real-valued functions, $\mathbb{P}$ acts on the space of vector-valued functions $f: V \rightarrow \mathbf{R}^{d}$.

Theorem 2.4. Suppose $\mathbb{G}$ is consistent. Then for every positive integer $t$, vertex $u \in V$, and function $\widehat{s}: V \rightarrow \mathbf{R}^{d}$ satisfying $\widehat{s}(v)=0$ for all $v \in V \backslash\{u\}$, we have $\|\widehat{s}(u)\|_{2}=\sum_{v}\left\|\widehat{s} \mathbb{P}^{t}(v)\right\|_{2}$.

Proof. The proof of this theorem is straightforward from the assumption that $\mathbb{G}$ is consistent. For $\widehat{p}=\widehat{s} \mathbb{P}^{t}$, note that $\widehat{p}(v)$ is the summation of all $d$-dimensional vectors resulting from rotating $\widehat{s}(u)$ via rotations along all possible paths of length $t$ from $u$ to $v$. Since $\mathbb{G}$ is consistent, the rotated vectors arriving at $v$ via different paths are positive multiples of the same vector. Also, the rotations maintain the 2-norm of vectors. Thus,

$$
\frac{\|\widehat{p}(v)\|_{2}}{\|\widehat{s}(u)\|_{2}}
$$

is simply the probability that a random walk in $G$ arrives at $v$ from $u$ after $t$ steps. The theorem follows. 


\section{PageRank Vectors in a Connection Graph}

The PageRank vector is based on random walks. Here we consider a lazy walk on $G$ with transition probability matrix $Z=(I+P) / 2$. In [Andersen et al. 06], a PageRank vector $\operatorname{pr}_{\alpha, s}$ is defined by a recurrence relation involving a seed vector $s$ (as a probability distribution) and a positive jumping constant $\alpha<1$ (or transportation constant). Namely, $\operatorname{pr}_{\alpha, s}=\alpha s+\operatorname{pr}_{\alpha, s}(1-\alpha) Z$.

For the connection graph $\mathbb{G}$, the PageRank vector $\widehat{\mathrm{pr}}_{\alpha, \widehat{s}}: V \rightarrow \mathbf{R}^{d}$ is defined by the same recurrence relation involving a seed vector $\widehat{s}: V \rightarrow \mathbf{R}^{d}$ and a positive jumping constant $\alpha<1$ :

$$
\widehat{\mathrm{pr}}_{\alpha, \widehat{s}}=\alpha \widehat{s}+(1-\alpha) \widehat{\mathrm{pr}}_{\alpha, \widehat{s}} \mathbb{Z},
$$

where $\mathbb{Z}=\frac{1}{2}\left(I_{n d \times n d}+\mathbb{P}\right)$ is the transition probability matrix of a lazy random walk on $\mathbb{G}$. An alternative definition of the PageRank vector is the following geometric sum of random walks:

$$
\widehat{\mathrm{pr}}_{\alpha, \widehat{s}}=\alpha \sum_{t=0}^{\infty}(1-\alpha)^{t} \widehat{s} \mathbb{Z}^{t}=\alpha \widehat{s}+(1-\alpha) \widehat{\mathrm{pr}}_{\alpha, \widehat{s} \mathbb{Z}} \cdot
$$

By Theorem 2.4 and (3.1), we obtain the following useful fact concerning PageRank vectors for a consistent connection graph.

Proposition 3.I. Suppose that a connection graph $\mathbb{G}$ is consistent. Then for every $u \in V, \alpha \in(0,1)$, and function $\widehat{s}: V \rightarrow \mathbf{R}^{d}$ satisfying $\|\widehat{s}(u)\|_{2}=1$ and $\widehat{s}(v)=0$ for $v \neq u$, we have $\left\|\widehat{\operatorname{pr}}_{\alpha, \widehat{s}}(v)\right\|_{2}=\operatorname{pr}_{\alpha, \chi_{u}}(v)$. Here $\chi_{u}: V \rightarrow \mathbb{R}$ denotes the characteristic function for the vertex $u$, so $\chi_{u}(v)=1$ for $v=u$, and $\chi_{u}(v)=0$ otherwise. In particular, $\sum_{v \in V}\left\|\widehat{p r}_{\alpha, \widehat{s}}(v)\right\|_{2}=\left\|\operatorname{pr}_{\alpha, \chi_{u}}\right\|_{1}=1$.

Proof. Since the function $\widehat{s}$ satisfies $\|\widehat{s}(u)\|_{2}=1$ and $\widehat{s}(v)=0$ for $v \neq u$, by Theorem 2.4, for a fixed $v \in V$, we conclude that $\left[\widehat{s} \mathbb{Z}^{t}\right](v)$ assumes a single value for all $t>0$. By the geometric sum expression of the PageRank vector, we have

$$
\begin{aligned}
\left\|\widehat{\mathrm{pr}}_{\alpha, \widehat{s}}(v)\right\|_{2} & =\left\|\alpha \sum_{t=0}^{\infty}(1-\alpha)^{t}\left[\widehat{s} \mathbb{Z}^{t}\right](v)\right\|_{2}=\alpha \sum_{t=0}^{\infty}(1-\alpha)^{t}\left\|\left[\widehat{s} \mathbb{Z}^{t}\right](v)\right\|_{2} \\
& =\alpha \sum_{t=0}^{\infty}(1-\alpha)^{t}\left[\chi_{u} Z^{t}\right](v)=\operatorname{pr}_{\alpha, \chi_{u}}(v) .
\end{aligned}
$$

Thus,

$$
\sum_{v \in V}\left\|\widehat{p r}_{\alpha, \widehat{s}}(v)\right\|_{2}=\left\|\operatorname{pr}_{\alpha, \chi_{u}}\right\|_{1}=1
$$




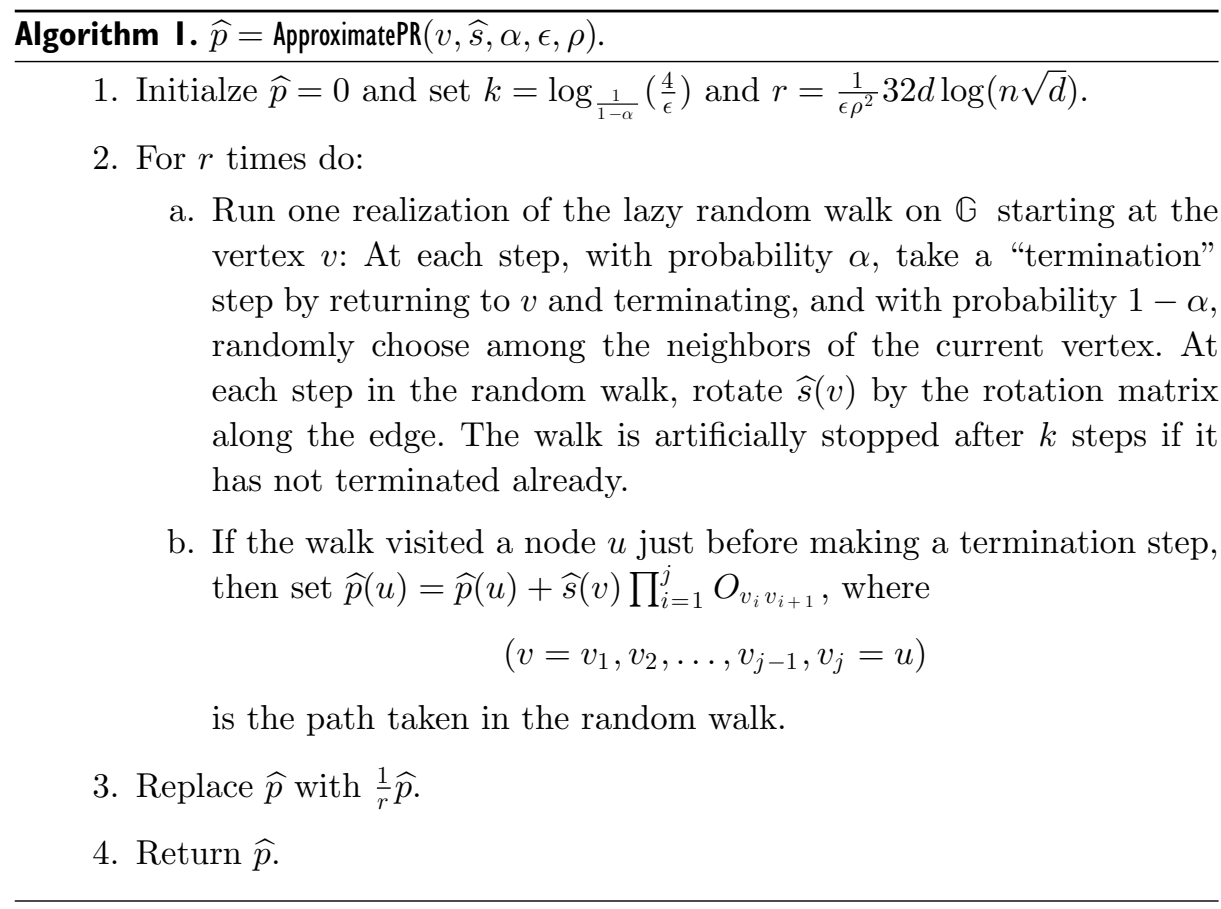

We will call such a PageRank vector $\widehat{\mathrm{pr}}_{\alpha, \widehat{s}}$ a connection PageRank vector on $u$. We next examine the problem of efficiently computing connection PageRank vectors. For graphs, an efficient sublinear algorithm is given in [Borgs et al. 12], in which PageRank vectors are approximated by realizing random walks of some bounded length. We here develop a version, our Algorithm 1, of their algorithm to apply to connection graphs. Our proof follows the template of their analysis, but uses the connection random walk.

For our analysis of the algorithm, we will need the following well-known concentration inequalities.

Lemma 3.2. (Multiplicative Chernoff bounds.) Let $X_{i}$ be i.i.d. Bernoulli random variables each with expectation $\mu$. Define $X=\sum_{i=1}^{n} X_{i}$. Then:

- For $0<\lambda<1, \operatorname{Pr}(X<(1-\lambda) \mu n)<\exp \left(-\mu n \lambda^{2} / 2\right)$.

- For $0<\lambda<1, \operatorname{Pr}(X>(1+\lambda) \mu n)<\exp \left(-\mu n \lambda^{2} / 4\right)$.

- For $\lambda \geq 1, \operatorname{Pr}(X>(1+\lambda) \mu n)<\exp (-\mu n \lambda / 2)$. 
Theorem 3.3. Let $\mathbb{G}=(V, E, O, w)$ be a connection graph and fix a vertex $v \in V$. Let $0<\epsilon<1$ be an additive error parameter, $0<\rho<1$ a multiplicative approximation parameter, and $0<\alpha<1$ a teleportation probability. Let $\widehat{s}: V \rightarrow \mathbf{R}^{d}$ be a function satisfying $\|\widehat{s}(v)\|_{2}=1$ and $\widehat{s}(u)=0$ for $u \neq v$. Then with probability at least $1-\Theta\left(\frac{1}{n^{2}}\right)$, Algorithm 1 produces a vector $\widehat{p}$ that satisfies

$$
\left\|\widehat{p}(u)-\widehat{\mathrm{pr}}_{\alpha, \widehat{s}}(u)\right\|_{2}<\rho\left\|\widehat{\mathrm{pr}}_{\alpha, \widehat{s}}(u)\right\|_{2}+\frac{\epsilon}{4}
$$

for vertices $u$ of $V$ for which $\left\|\widehat{p r}_{\alpha, \widehat{s}}(u)\right\|_{2} \geq \frac{\epsilon}{4}$, and satisfying $\|\widehat{p}(u)\|_{2}<\frac{\epsilon}{2}$ for vertices $u$ for which $\left\|\widehat{p r}_{\alpha, \widehat{s}}(u)\right\|_{2} \leq \frac{\epsilon}{4}$. The running time of the algorithm is

$$
O\left(\frac{d^{3} \log (n \sqrt{d}) \log (1 / \epsilon)}{\epsilon \rho^{2} \log (1 /(1-\alpha))}\right)
$$

Proof. We have from (3.1) that

$$
\widehat{\operatorname{pr}}_{\alpha, \widehat{s}}=\alpha \widehat{s} \sum_{t=0}^{\infty}(1-\alpha)^{t} \mathbb{Z}^{t} .
$$

We observe that the $t$ th term in this sum is the contribution to the PageRank vector given by the walks of length $t$. We will approximate this by looking at walks of length at most $k$. Define

$$
\widehat{p}_{\alpha, \widehat{s}}^{(k)}=\alpha \widehat{s} \sum_{t=0}^{k}(1-\alpha)^{t} \mathbb{Z}^{t} .
$$

We then observe that by choosing $k$ large enough that $(1-\alpha)^{k}<\epsilon / 4$, we have

$$
\left\|\widehat{p r}_{\alpha, \widehat{s}}-\widehat{p}_{\alpha, \widehat{s}}^{(k)}\right\|_{2}<\frac{\epsilon}{4} .
$$

The choice of $k=\log _{\frac{1}{1-\alpha}}\left(\frac{4}{\epsilon}\right)$ will guarantee this.

The output $\widehat{p}$ of the algorithm gives an approximation to $\widehat{p}_{\alpha, \widehat{s}}^{(k)}$ by realizing walks of length at most $k$. The algorithm does so by taking the average count over $32 d \log (n \sqrt{d}) / \epsilon \rho^{2}$ trials. Note that $\widehat{p}_{\alpha, \widehat{s}}^{(k)}(u)$ is the expected value of the contribution of an instance of a random walk of length $k$. We will take an arbitrary entry of $\widehat{p}(u)$, say $\widehat{p}(u)(j)$, and compare it to $\widehat{p}_{\alpha, \widehat{s}}^{(k)}(u)(j)$. Assuming that for at least one $j$, we have $\widehat{p}_{\alpha, \widehat{s}}^{(k)}(u)(j)>\epsilon / 4 d$, we get by the multiplicative Chernoff bound that

$$
\operatorname{Pr}\left(\widehat{p}(u)(j)<(1+\rho) \widehat{p}_{\alpha, \widehat{s}}^{(k)}(u)(j)\right)<\exp (-2 \log (n \sqrt{d}))
$$


and

$$
\operatorname{Pr}\left(\widehat{p}(u)(j)<(1-\rho) \widehat{p}_{\alpha, \widehat{s}}^{(k)}(u)(j)\right)<\exp (-2 \log (n \sqrt{d}))
$$

which implies

$$
\operatorname{Pr}\left(\left|\widehat{p}(u)(j)-\widehat{p}_{\alpha, \widehat{s}}^{(k)}(u)(j)\right|>\rho \widehat{p}_{\alpha, \widehat{s}}^{(k)}(u)(j)\right)<2 \exp (-2 \log (n \sqrt{d})) .
$$

Note that this difference will be the same for all the entries of $\widehat{p}(u)$, and therefore,

$$
\operatorname{Pr}\left(\left\|\widehat{p}(u)-\widehat{p}_{\alpha, \widehat{s}}^{(k)}(u)\right\|_{2}>\rho\left\|\widehat{p}_{\alpha, \widehat{s}}^{(k)}(u)\right\|_{2}\right)<2 d \exp (-2 \log (n \sqrt{d}))=\frac{2}{n^{2}} .
$$

In a similar manner, if $\widehat{p}_{\alpha, \widehat{s}}^{(k)}(u)(j) \leq \epsilon / 4 d$, then by the Chernoff bound, we have

$$
\operatorname{Pr}\left(\widehat{p}(u)(j)>\frac{\epsilon}{2 d}\right)<\exp (-2 \log (n \sqrt{d}))
$$

so

$$
\operatorname{Pr}\left(\|\widehat{p}(u)\|_{2}>\frac{\epsilon}{2}\right)<d \exp (-2 \log (n \sqrt{d}))=\frac{1}{n^{2}} .
$$

For the running time, note that the algorithm performs $32 d \log (n \sqrt{d}) / \epsilon \rho^{2}$ rounds, where each round simulates a walk of length at most $\log _{\frac{1}{1-\alpha}}\left(\frac{4}{\epsilon}\right)$, each walk multiplying $\widehat{s}(v)$ by the $d \times d$ rotation matrices. Thus the running time is

$$
O\left(\frac{d^{3} \log (n \sqrt{d}) \log (1 / \epsilon)}{\epsilon \rho^{2} \log (1 /(1-\alpha))}\right),
$$

which completes the proof

\section{The Connection Resistance}

Motivated by the definition of effective resistance in electrical network theory, we consider the block matrix

$$
\Psi=\mathbb{B} \mathbb{L}_{\mathbb{G}}^{+} \mathbb{B}^{T} \in \mathcal{F}(m, m, d ; \mathbf{R}),
$$

where $\mathbb{L}^{+}$is the pseudoinverse of $\mathbb{L}$. Note that for a matrix $M$, the pseudoinverse of $M$ is defined as the unique matrix $M^{+}$satisfying the following four criteria [Golub and Van Loan 96, Penrose 55]:

(i) $M M^{+} M=M$;

(ii) $M^{+} M M^{+}=M^{+}$;

(iii) $\left(M M^{+}\right)^{*}=\left(M M^{+}\right)$; 
(iv) $\left(M^{+} M\right)^{*}=M^{+} M$.

We define the connection resistance $\mathbb{R}_{\text {eff }}(e)$ as $\mathbb{R}_{\text {eff }}(v, u)=\|\Psi(e, e)\|_{2}$. Note that block $\Psi(e, e)$ is a $d \times d$ matrix. We will show that in the case that the connection graph $\mathbb{G}$ is consistent, $\mathbb{R}_{\text {eff }}(u, v)$ is reduced to the usual effective resistance $R_{\text {eff }}(u, v)$ of the underlying graph $G$. In general, if the connection graph is not consistent, the connection resistance is not necessarily equal to its effective resistance in the underlying graph $G$.

Our first observation is the following lemma.

Lemma 4.I. Suppose $\mathbb{G}$ is a consistent connection graph whose underlying graph is connected. For two vertices $u, v$ of $\mathbb{G}$, let $p_{u v}=\left(v_{1}=u, v_{2}, \ldots, v_{k}=v\right)$ be any path from $u$ to $v$ in $\mathbb{G}$. Define $O_{p_{u v}}=\prod_{j=1}^{k-1} O_{v_{j} v_{j+1}}$. Let $\mathbb{R}$ be the connection Laplacian of $\mathbb{G}$ and let $L$ be the discrete Laplacian of $G$ respectively. Then

$$
\mathbb{L}^{+}(u, v)= \begin{cases}L^{+}(u, v) O_{p_{u v}}, & i \neq j, \\ L^{+}(u, v) I_{d \times d}, & i=j .\end{cases}
$$

Proof. We first note that the matrix $O_{p_{u v}}$ is well defined, since $G$ is consistent. Also note that if $u$ and $v$ are adjacent, then $O_{p_{u v}}=O_{u v}$. Also observe that $\mathbb{L}(u, v)=L(u, v) O_{p_{u v}}$, since if $u v$ is not an edge, $L(u, v)=0$, and if $u, v$ is an edge, $O_{p_{u v}}=O_{u v}$. To verify that $\mathbb{L}^{+}$is the pseudoinverse of $\mathbb{L}$, we just need to verify that $\mathbb{L}^{+}(u, v)$ satisfies all four criteria above.

To see (i) $\mathbb{L}^{+} \mathbb{L}=\mathbb{L}$, we consider two vertices $u$ and $v$ and note that

$$
\begin{aligned}
\left(\mathbb{L} \mathbb{L}^{+} \mathbb{L}\right)(u, v) & =\sum_{x, y} \mathbb{L}(u, x) \mathbb{L}^{+}(x, y) \mathbb{L}(y, v) \\
& =\sum_{x, y} L(u, x) L^{+}(x, y) L(y, v) O_{p_{u x}} O_{p_{x y}} O_{p_{y v}} \\
& =\sum_{x, y} L(u, x) L^{+}(x, y) L(y, v) O_{p_{u v}},
\end{aligned}
$$

where the last equality follows by consistency. Since $L^{+}$is the pseudoinverse of $L$, we also have $L L^{+} L=L$, which implies that

$$
L(u, v)=\sum_{x, y} L(u, x) L^{+}(x, y) L(y, v) .
$$

Thus,

$$
\left(\mathbb{L} \mathbb{L}^{+} \mathbb{L}\right)(u, v)=L(u, v) O_{p_{u v}}=\mathbb{L}(u, v),
$$

and the verification of (i) is complete. 
The verification of (ii) is quite similar to that of (i), and we omit it here.

To see (iii) $\left(\mathbb{L} \mathbb{L}^{+}\right)^{*}=\left(\mathbb{L} \mathbb{L}^{+}\right)$, we also consider two fixed vertices $v_{i}$ and $v_{j}$. Note that

$$
\begin{aligned}
\left(\mathbb{L} \mathbb{L}^{+}\right)(u, v) & =\sum_{x} \mathbb{L}(u, x) \mathbb{L}^{+}(x, v)=\sum_{x} L(u, x) L^{+}(x, v) O_{p_{u x}} O_{p_{x v}} \\
& =\sum_{x} L(u, x) L^{+}(x, v) O_{p_{u v}} .
\end{aligned}
$$

On the other hand,

$$
\left(\mathbb{L} \mathbb{L}^{+}\right)(v, u)=\sum_{x} L(v, x) L^{+}(x, u) O_{p_{v u}}=\sum_{x} L(v, x) L^{+}(x, u) O_{p_{u v}}^{T} .
$$

Since $L^{+}$is the pseudoinverse of $L$, we also have $\left(L L^{+}\right)^{*}=L L^{+}$, which implies that

$$
\sum_{x} L(u, x) L^{+}(x, v)=\sum_{x} L(v, x) L^{+}(x, u)
$$

and thus $\left(\mathbb{L} \mathbb{L}^{+}\right)^{*}=\left(\mathbb{L} \mathbb{L}^{+}\right)$.

The verification of (iv) $\left(\mathbb{L}^{+} \mathbb{L}\right)^{*}=\mathbb{L}^{+} \mathbb{L}$ is also similar to (iii). This completes the proof of the lemma.

Using the above lemma, we examine the relation between the connection resistance and the effective resistance for a consistent connection graph by the following theorem.

Theorem 4.2. Suppose $\mathbb{G}=(V, E, O, w)$ is a consistent connection graph whose underlying graph $G$ is connected. Then for every edge $(u, v) \in \mathbb{G}$, we have

$$
\mathbb{R}_{\text {eff }}(u, v)=R_{\text {eff }}(u, v) .
$$

Proof. Let $\mathbb{L}$ be the connection Laplacian of $\mathbb{G}$, and $L$ the Laplacian of the underlying graph $G$. Let us fix an edge $e=(u, v) \in \mathbb{G}$. By the definition of effective resistance, $R_{\text {eff }}(u, v)$ is the maximum eigenvalue of the matrix

$$
\Psi(e, e)=\left[\begin{array}{ll}
O_{v u}-I_{d \times d}
\end{array}\right]\left[\begin{array}{l}
\mathbb{L}^{+}(u, u) \mathbb{L}^{+}(u, v) \\
\mathbb{L}^{+}(v, u) \mathbb{L}^{+}(v, v)
\end{array}\right]\left[\begin{array}{c}
O_{u v} \\
-I_{d \times d}
\end{array}\right],
$$

where $O_{u v}$ is the rotation from $u$ to $v$. By Lemma 4.1, we have

$$
\begin{aligned}
& \mathbb{L}^{+}(u, u)=L^{+}(u, u) I_{d \times d}, \quad \mathbb{L}^{+}(u, v)=L^{+}(u, v) O_{p_{u v}}, \\
& \mathbb{L}^{+}(v, v)=L^{+}(v, v) I_{d \times d}, \quad \mathbb{L}^{+}(v, u)=L^{+}(v, u) O_{p_{v u}}=L^{+}(u, v) O_{p_{v u}} .
\end{aligned}
$$


Algorithm 2. $(\widetilde{\mathbb{G}}=(V, \widetilde{E}, O, \widetilde{w}))=\operatorname{Sample}\left(\mathbb{G}=(V, E, O, w), p^{\prime}, q\right)$.

1. For every edge $e \in E$, set $p_{e}$ proportional to $p_{e}^{\prime}$.

2. Choose a random edge $e$ of $\mathbb{G}$ with probability $p_{e}$, and add $e$ to $\widetilde{\mathbb{G}}$ with edge weight $\widetilde{w}_{e}=w_{e} / q p_{e}$. Take $q$ samples independently with replacement, summing weights if an edge is chosen more than once.

3. Return $\widetilde{G}$.

Thus, by the definition of the matrix $\Psi$,

$$
\Psi(e, e)=\left(L_{u, u}^{+}+L_{v, v}^{+}\right) I_{d \times d}-L_{u, v}^{+}\left(O_{p_{v u}} O_{u v}+O_{v u} O_{p_{u v}}\right) .
$$

Note that $O_{p_{u v}} O_{v u}=O_{u v} O_{u v}^{T}=I$, and similarly, $O_{v u} O_{p_{v u}}=I$, so

$$
\Psi(e, e)=\left(L^{+}(u, u)+L^{+}(v, v)-2 L^{+}(u, v)\right) I_{d \times d} .
$$

Note that $\left(L^{+}(u, u)+L^{+}(v, v)-2 L^{+}(u, v)\right)$ is exactly the effective resistance of $e$, so

$$
\|\Psi(e, e)\|_{2}=L^{+}(u, u)+L^{+}(v, v)-2 L^{+}(u, v)=R_{\mathrm{eff}}(u, v) .
$$

The theorem is proved.

\section{Ranking Edges Using the Connection Resistance}

A central part of a graph-sparsification algorithm is the sampling technique for selecting edges. It is crucial to choose the appropriate probabilistic distribution that can lead to a sparsifier preserving every cut in the original graph. In [Spielman and Srivastava 08], the measure of how well the sparsifier preserves the cuts is given according to how well the sparsifier preserves the spectral properties of the original graph. We follow the template of [Spielman and Srivastava 08] to present a sampling algorithm that will accomplish this. Algorithm 2 is a generic sampling algorithm for a graph-sparsification problem. We will sample edges using the distribution proportional to the weighted connection resistances.

Theorem 5.I. For a given connection graph $\mathbb{G}$ and some positive $\xi>0$, we consider $\widetilde{\mathbb{G}}=\operatorname{Sample}\left(G, p^{\prime}, q\right)$, where $p_{e}^{\prime}=w_{e} \mathbb{R}_{\text {eff }}(e)$ and

$$
q=\frac{4 n d(\log (n d)+\log (1 / \xi))}{\epsilon^{2}} .
$$


Suppose $\mathbb{G}$ and $\widetilde{\mathbb{G}}$ have connection Laplacians $\mathbb{L}_{\mathbb{G}}$ and $\mathbb{L}_{\widetilde{\mathbb{G}}}$ respectively. Then with probability at least $1-\xi$, for every function $f: V \rightarrow \mathbf{R}^{d}$, we have

$$
(1-\epsilon) f \mathbb{L}_{\mathbb{G}} f^{T} \leq f \mathbb{L}_{\widetilde{G}} f^{T} \leq(1+\epsilon) f \mathbb{L}_{\mathbb{G}} f^{T} .
$$

Before proving Theorem 5.1, we need the following two lemmas, in particular concerning the matrix $\Lambda=\mathbb{W}^{1 / 2} \mathbb{B} \mathbb{L}_{\mathbb{G}}^{+} \mathbb{B}^{T} \mathbb{W}^{1 / 2}$.

Lemma 5.2. The matrix $\Lambda$ is a projection matrix, i.e., $\Lambda^{2}=\Lambda$.

Proof. Observe that

$$
\begin{aligned}
\Lambda^{2} & =\left(\mathbb{W}^{1 / 2} \mathbb{B} \mathbb{L}_{\mathbb{G}}^{+} \mathbb{B}^{T} \mathbb{W}^{1 / 2}\right)\left(\mathbb{W}^{1 / 2} \mathbb{B} \mathbb{L}_{\mathbb{G}}^{+} \mathbb{B}^{T} \mathbb{W}^{1 / 2}\right) \\
& =\mathbb{W}^{1 / 2} \mathbb{B} \mathbb{L}_{\mathbb{G}}^{+} \mathbb{L}_{\mathbb{G}} \mathbb{L}_{\mathbb{G}}^{+} \mathbb{B}^{T} \mathbb{W}^{1 / 2}=\mathbb{W}^{1 / 2} \mathbb{B} \mathbb{L}_{\mathbb{G}}^{+} \mathbb{B}^{T} \mathbb{W}^{1 / 2}=\Lambda .
\end{aligned}
$$

The lemma follows.

To show that $\widetilde{\mathbb{G}}=(V, \widetilde{E}, O, \widetilde{w})$ is a good sparsifier for $\mathbb{G}$ satisfying (5.1), we need to show that the quadratic forms $f \mathbb{L}_{\widetilde{G}} f^{T}$ and $f \mathbb{L}_{\mathbb{G}} f^{T}$ are close. By applying similar methods as in [Spielman and Srivastava 08], we reduce the problem of preserving $f \mathbb{L}_{\mathbb{G}} f^{T}$ to that of $g \Lambda g^{T}$ for some function $g$. We consider a diagonal matrix $\mathbb{S} \in \mathcal{F}(m, m, d ; \mathbf{R})$, where the diagonal blocks are scalar matrices given by

$$
\mathbb{S}(e, e)=\frac{\widetilde{w}_{e}}{w_{e}} I_{d \times d}=\frac{N_{e}}{q p_{e}} I_{d \times d},
$$

and $N_{e}$ is the number of times an edge $e$ is sampled.

Lemma 5.3. Suppose $\mathbb{S}$ is a nonnegative diagonal matrix such that

$$
\|\Lambda \Im \Lambda-\Lambda \Lambda\|_{2} \leq \epsilon
$$

Then

$$
\forall f: V \rightarrow \mathbf{R}^{d}, \quad(1-\epsilon) f \mathbb{L}_{\mathbb{G}} f^{T} \leq f \mathbb{L}_{\widetilde{G}} f^{T} \leq(1+\epsilon) f \mathbb{L}_{\mathbb{G}} f^{T},
$$

where $\mathbb{L}_{\widetilde{\mathbb{G}}}=\mathbb{B}^{T} \mathbb{W}^{1 / 2} \mathbb{S} \mathbb{W}^{1 / 2} \mathbb{B}$.

Proof. The assumption is equivalent to

$$
\sup _{f \in \mathbf{R}^{m d}, f \neq 0} \frac{\left|f \Lambda(\mathbb{S}-I) \Lambda f^{T}\right|}{f f^{T}} \leq \epsilon .
$$


Restricting our attention to vectors in $\operatorname{Im}\left(\mathbb{B}^{T} \mathbb{W}^{1 / 2}\right)$, we have

$$
\sup _{f \in \operatorname{Im}\left(\mathbb{B}^{T} W^{1 / 2}\right), f \neq 0} \frac{\left|f \Lambda(\mathbb{S}-I) \Lambda f^{T}\right|}{f f^{T}} \leq \epsilon .
$$

Since $\Lambda$ is the identity on $\operatorname{Im}\left(\mathbb{B}^{T} W^{1 / 2}\right)$, we have $f \Lambda=f$ for all $f \in$ $\operatorname{Im}\left(\mathbb{B}^{T} \mathbb{W}^{1 / 2}\right)$. Also, every such $f$ can be written as $f=g \mathbb{B}^{T} \mathbb{W}^{1 / 2}$ for $g \in \mathbf{R}^{n d}$. Thus

$$
\begin{aligned}
\sup _{f \in \operatorname{Im}\left(\mathbb{B}^{T} \mathbb{W}^{1 / 2}\right)} \frac{\left|f \Lambda(\mathbb{S}-I) \Lambda f^{T}\right|}{f \neq 0}=\sup _{\substack{f \in \operatorname{Im}\left(\mathbb{B}^{T} \mathbb{W}^{1 / 2}\right) \\
f \neq 0}} \frac{\left|f(\mathbb{S}-I) f^{T}\right|}{f f^{T}} \\
=\sup _{\substack{g \in \mathbf{R}^{n d} \\
g \mathbb{B}^{T} W^{1 / 2} \neq 0}} \frac{\left|g \mathbb{B}^{T} \mathbb{W}^{1 / 2} \mathbb{S} \mathbb{W}^{1 / 2} \mathbb{B} g^{T}-g \mathbb{B}^{T} \mathbb{W} \mathbb{B} g^{T}\right|}{g \mathbb{B}^{T} \mathbb{W} g^{T}} \\
=\sup _{\substack{g \in \mathbf{R}^{n d} \\
g \mathbb{B}^{T} \mathbb{W}^{1 / 2} \neq 0}} \frac{\left|g \mathbb{L}_{\widetilde{\mathbb{G}}} g^{T}-g \mathbb{L}_{\mathbb{G}} g^{T}\right|}{g \mathbb{L}_{\mathbb{G}} g^{T}} \leq \epsilon .
\end{aligned}
$$

Rearranging yields the desired conclusion for all $g \in \mathbf{R}^{n d}$.

We also require the following concentration inequality in order to prove our main theorems. Various matrix concentration inequalities have been derived; see [Achlioptas 01, Cristofides and Markström 08, Recht 11, Tropp 11]. Here we will use the simple version that is proved in [Vershynin 08].

Theorem 5.4. Let $X_{1}, X_{2}, \ldots, X_{q}$ be independent symmetric random $k \times k$ matrices with zero means, $S_{q}=\sum_{i} X_{i},\left\|X_{i}\right\|_{2} \leq 1$ for all $i$ a.s. Then for every $t>0$, we have

$$
\operatorname{Pr}\left[\left\|S_{q}\right\|_{2}>t\right] \leq k \max \left(\exp \left(-\frac{t^{2}}{4 \sum_{i}\left\|\operatorname{Var}\left(X_{i}\right)\right\|_{2}}\right), \exp \left(-\frac{t}{2}\right)\right) .
$$

A direct consequence of Theorem 5.4 is the following corollary.

Corollary 5.5. Suppose $X_{1}, X_{2}, \ldots, X_{q}$ are independent random symmetric $k \times k$ matrices satisfying

(1) for all $1 \leq i \leq q,\left\|X_{i}\right\|_{2} \leq M$ a.s.,

(2) for all $1 \leq i \leq q,\left\|\operatorname{Var}\left(X_{i}\right)\right\|_{2} \leq M\left\|\mathbf{E}\left[X_{i}\right]\right\|_{2}$. 
Then for every $\epsilon \in(0,1)$, we have

$$
\operatorname{Pr}\left[\left\|\sum_{i} X_{i}-\sum_{i} \mathbf{E}\left[X_{i}\right]\right\|_{2}>\epsilon \sum_{i}\left\|\mathbf{E}\left[X_{i}\right]\right\|_{2}\right] \leq k \exp \left(-\frac{\epsilon^{2} \sum_{i}\left\|\mathbf{E}\left[X_{i}\right]\right\|_{2}}{4 M}\right) .
$$

Proof. Let us consider the independent random symmetric matrices

$$
\frac{X_{i}-\mathbf{E}\left[X_{i}\right]}{M}
$$

for $1 \leq i \leq q$. Clearly, they are independent symmetric random $k \times k$ matrices with zero means satisfying

$$
\left\|\frac{X_{i}-\mathbf{E}\left[X_{i}\right]}{M}\right\|_{2} \leq 1
$$

for $1 \leq i \leq q$. We note also that

$$
\operatorname{Var}\left(\frac{X_{i}-\mathbf{E}\left[X_{i}\right]}{M}\right)=\operatorname{Var}\left(\frac{X_{i}}{M}\right)=\frac{\operatorname{Var}\left(X_{i}\right)}{M^{2}} .
$$

Thus by applying Theorem 5.4, we have

$$
\begin{aligned}
\operatorname{Pr} & {\left[\left\|\frac{\sum_{i} X_{i}-\mathbf{E}\left[X_{i}\right]}{M}\right\|_{2}>t\right]=\operatorname{Pr}\left[\left\|\sum_{i} X_{i}-\sum_{i} \mathbf{E}\left[X_{i}\right]\right\|_{2}>t M\right] } \\
& \leq k \max \left(\exp \left(-\frac{t^{2} M^{2}}{4 \sum_{i}\left\|\operatorname{Var}\left(X_{i}\right)\right\|_{2}}\right), \exp \left(-\frac{t}{2}\right)\right) .
\end{aligned}
$$

Note that by condition (2), we obtain

$$
\sum_{i}\left\|\operatorname{Var}\left(X_{i}\right)\right\|_{2} \leq M \sum_{i}\left\|\mathbf{E}\left[X_{i}\right]\right\|_{2} .
$$

Thus if we set

$$
t=\frac{\epsilon \sum_{i}\left\|\mathbf{E}\left[X_{i}\right]\right\|_{2}}{M},
$$

the left term on the right-hand side of (5.2) can be bounded as follows:

$$
\frac{t^{2} M^{2}}{4 \sum_{i=1}^{q}\left\|\operatorname{Var}\left(X_{i}\right)\right\|_{2}} \geq \frac{\left(\epsilon \sum_{i=1}^{q}\left\|\mathbf{E}\left[X_{i}\right]\right\|_{2}\right)^{2}}{4 M \sum_{i=1}^{q}\left\|\mathbf{E}\left[X_{i}\right]\right\|_{2}}=\frac{\epsilon^{2} \sum_{i=1}^{q}\left\|\mathbf{E}\left[X_{i}\right]\right\|_{2}}{4 M}
$$

The corollary follows.

Proof of Theorem 5.I. Our algorithm samples edges from $\mathbb{G}$ independently with replacement, with probabilities $p_{e}$ proportional to $w_{e} \mathbb{R}_{\text {eff }}(e)$. Note that sampling 
$q$ edges from $\mathbb{G}$ corresponds to sampling $q$ columns from $\Lambda$. So we can write

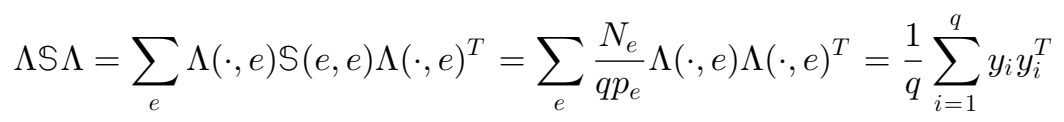

for block matrices $y_{1}, \ldots, y_{q} \in R^{n d \times d}$ drawn independently with replacement from the distribution $y=\frac{1}{\sqrt{p_{e}}} \Lambda(\cdot, e)$ with probability $p_{e}$. Now we can apply Corollary 5.5. The expectation of $y y^{T}$ is given by

$$
\mathbf{E}\left[y y^{T}\right]=\sum_{e} p_{e} \frac{1}{p_{e}} \Lambda(\cdot, e) \Lambda(\cdot, e)^{T}=\Lambda,
$$

which implies that $\left\|\mathbf{E}\left[y y^{T}\right]\right\|_{2}=\|\Lambda\|_{2}=1$. We also have a bound on the norm of $y_{i} y_{i}^{T}$ :

$$
\left\|y_{i} y_{i}^{T}\right\|_{2} \leq \max _{e}\left(\frac{\left\|\Lambda(\cdot, e)^{T} \Lambda(\cdot, e)\right\|_{2}}{p_{e}}\right)=\max _{e}\left(\frac{w_{e} \mathbb{R}_{\text {eff }}(e)}{p_{e}}\right) .
$$

Since the probability $p_{e}$ is proportional to $w_{e} \mathbb{R}_{\text {eff }}(e)$, i.e.,

$$
p_{e}=\frac{w_{e} \mathbb{R}_{\text {eff }}(e)}{\sum_{e} w_{e} \mathbb{R}_{\text {eff }}(e)}=\frac{\|\Lambda(e, e)\|_{2}}{\sum_{e}\|\Lambda(e, e)\|_{2}},
$$

we have

$$
\left\|y_{i} y_{i}^{T}\right\|_{2} \leq \sum_{e}\|\Lambda(e, e)\|_{2} \leq \sum_{e} \operatorname{Tr}(\Lambda(e, e))=\operatorname{Tr}(\Lambda) \leq n d
$$

To bound the variance, observe that

$$
\begin{aligned}
\left\|\operatorname{Var}\left(y y^{T}\right)\right\|_{2} & =\left\|\mathbf{E}\left[y y^{T} y y^{T}\right]-\left(\mathbf{E}\left[y y^{T}\right]\right)^{2}\right\|_{2} \\
& \leq\left\|\mathbf{E}\left[y y^{T} y y^{T}\right]\right\|_{2}+\left\|\left(\mathbf{E}\left[y y^{T}\right]\right)^{2}\right\|_{2} .
\end{aligned}
$$

Since the second term on the right-hand of the above inequality can be bounded by

$$
\left\|\left(\mathbf{E}\left[y y^{T}\right]\right)^{2}\right\|_{2}=\left\|\Lambda^{2}\right\|_{2}(\operatorname{as} \text { property }(1))=\|\Lambda\|_{2}=1,
$$

it is sufficient to bound the term $\left\|\mathbf{E}\left[y y^{T} y y^{T}\right]\right\|_{2}$. By the definition of expectation, we observe that

$$
\begin{aligned}
\left\|\mathbf{E}\left[y y^{T} y y^{T}\right]\right\|_{2} & =\left\|\sum_{e} p_{e} \frac{1}{p_{e}^{2}} \Lambda(\cdot, e) \Lambda(\cdot, e)^{T} \Lambda(\cdot, e) \Lambda(\cdot, e)^{T}\right\|_{2} \\
& =\left\|\sum_{e} \frac{1}{p_{e}} \Lambda(\cdot, e) \Lambda(e, e) \Lambda(\cdot, e)^{T}\right\|_{2}
\end{aligned}
$$


This implies that

$$
\begin{aligned}
& \left\|\mathbf{E}\left[y y^{T} y y^{T}\right]\right\|_{2} \\
& =\max _{f \in \operatorname{Im}\left(\mathbb{W}^{1 / 2} \mathbb{B}\right)} \sum_{e} \frac{1}{p_{e}} \frac{f^{T} \Lambda(\cdot, e) \Lambda(e, e) \Lambda(\cdot, e)^{T} f}{f^{T} f} \\
& =\max _{f \in \operatorname{Im}\left(\mathbb{W}^{1 / 2} \mathbb{B}\right)} \sum_{e} \frac{1}{p_{e}} \frac{f^{T} \Lambda(\cdot, e) \Lambda(e, e) \Lambda(\cdot, e)^{T} f}{f^{T} \Lambda(\cdot, e) \Lambda(\cdot, e)^{T} f} \frac{f^{T} \Lambda(\cdot, e) \Lambda(\cdot, e)^{T} f}{f^{T} f} \\
& \leq \max _{f \in \operatorname{Im}\left(\mathbb{W}^{1 / 2} \mathbb{B}\right)} \sum_{e} \frac{\|\Lambda(e, e)\|_{2}}{p_{e}} \frac{f^{T} \Lambda(\cdot, e) \Lambda(\cdot, e)^{T} f}{f^{T} f} .
\end{aligned}
$$

Recall that the probability $p_{e}$ is proportional to $w_{e} \mathbb{R}_{\text {eff }}(e)$, i.e.,

$$
p_{e}=\frac{w_{e} \mathbb{R}_{\text {eff }}(e)}{\sum_{e} w_{e} \mathbb{R}_{\text {eff }}(e)}=\frac{\|\Lambda(e, e)\|_{2}}{\sum_{e}\|\Lambda(e, e)\|_{2}} .
$$

We have

$$
\begin{aligned}
\left\|\mathbf{E}\left[y y^{T} y y^{T}\right]\right\|_{2} & \leq \sum_{e}\|\Lambda(e, e)\|_{2}\left(\max _{f \in \operatorname{Im}\left(\mathbb{W}^{1 / 2} \mathbb{B}\right)} \sum_{e} \frac{f^{T} \Lambda(\cdot, e) \Lambda(\cdot, e)^{T} f}{f^{T} f}\right) \\
& =\sum_{e}\|\Lambda(e, e)\|_{2}\|\Lambda\|_{2}=\sum_{e}\|\Lambda(e, e)\|_{2} \leq \sum_{e} \operatorname{Tr}(\Lambda(e, e)) \\
& =\operatorname{Tr}(\Lambda) \leq n d .
\end{aligned}
$$

Thus

$$
\left\|\operatorname{Var}\left(y y^{T}\right)\right\|_{2} \leq n d+1 \leq 2 n d\left\|\mathbf{E}\left[y y^{T}\right]\right\|_{2} .
$$

To complete the proof, setting $q=4 n d(\log (n d)+\log (1 / \xi)) / \epsilon^{2}$ and using the fact that the dimension of $y y^{T}$ is $n d$, we have

$$
\begin{aligned}
\operatorname{Pr}\left[\left\|\frac{1}{q} \sum_{i=1}^{q} y_{i} y_{i}^{T}-\mathbf{E}\left[y y^{T}\right]\right\|_{2}>\epsilon\right] & \leq n d \exp \left(-\frac{\epsilon^{2} \sum_{i=1}^{q}\left\|\mathbf{E}\left[y_{i} y_{i}^{T}\right]\right\|_{2}}{4 n d}\right) \\
& \leq n d \exp \left(-\frac{\epsilon^{2} q}{4 n d}\right) \leq \xi
\end{aligned}
$$

for some constant $0<\xi<1$. The theorem follows.

In [Koutis 10], a modification of the algorithm from [Spielman and Srivastava 08] is presented. The oversampling theorem in [Koutis 10] can further be modified for connection graphs and stated as follows.

Theorem 5.6. (0versampling.) For a given connection graph $\mathbb{G}$ and some positive $\xi>0$, we consider $\widetilde{\mathbb{G}}=\operatorname{Sample}\left(G, p^{\prime}, q\right)$, where $p_{e}^{\prime}=w_{e} \mathbb{R}_{\text {eff }}(e), t=\sum_{e \in E} p_{e}^{\prime}$, and $q=$ $4 t(\log (t)+\log (1 / \xi)) / \epsilon^{2}$. Suppose $\mathbb{G}$ and $\widetilde{\mathbb{G}}$ have respective connection Laplacians 
$\mathbb{L}_{\mathbb{G}}$ and $\mathbb{L}_{\widetilde{G}}$. Then with probability at least $1-\xi$, for all $f: V \rightarrow \mathbf{R}^{d}$, we have

$$
(1-\epsilon) f \mathbb{L}_{\mathbb{G}} f^{T} \leq f \mathbb{L}_{\widetilde{G}} f^{T} \leq(1+\epsilon) f \mathbb{L}_{\mathbb{G}} f^{T} .
$$

Proof. In the proof of Theorem 5.1, the key is the bound on the norm $\left\|y_{i} y_{i}^{T}\right\|_{2}$. If $p_{e}^{\prime} \geq w_{e} \mathbb{R}_{\text {eff }}(e)$, the norm $\left\|y_{i} y_{i}^{T}\right\|_{2}$ is bounded by $\sum_{e \in E} p_{e}^{\prime}$. The theorem follows.

Now let us consider a variation of the connection resistance denoted by $\overline{\mathbb{R}_{\text {eff }}}(e)=\operatorname{Tr}(\Psi(e, e))$. Clearly, we have

$$
\overline{\mathbb{R}_{\text {eff }}}(e)=\operatorname{Tr}(\Psi(e, e)) \geq\|\Psi(e, e)\|_{2}=\mathbb{R}_{\text {eff }}(e)
$$

and

$$
\sum_{e} w_{e} \overline{\mathbb{R}_{\text {eff }}}(e)=\sum_{e} \operatorname{Tr}(\Lambda(e, e))=\operatorname{Tr}(\Lambda) \leq n d .
$$

Using Theorem 5.6, we have the following.

Corollary 5.7. For a given connection graph $\mathbb{G}$ and some positive $\xi>0$, we consider $\widetilde{\mathbb{G}}=\operatorname{Sample}\left(G, p^{\prime}, q\right)$, where $p_{e}^{\prime}=w_{e} \overline{\mathbb{R}_{\text {eff }}}(e)$ and $q=4 n d(\log (n d)+\log (1 / \xi)) / \epsilon^{2}$. Suppose $\mathbb{G}$ and $\widetilde{\mathbb{G}}=\operatorname{Sample}\left(G, p^{\prime}, q\right)$ have respective connection Laplacians $\mathbb{L}_{\mathbb{G}}$ and $\mathbb{L}_{\widetilde{G}}$. Then with probability at least $1-\xi$, for all $f: V \rightarrow \mathbf{R}^{d}$, we have

$$
(1-\epsilon) f \mathbb{L}_{\mathbb{G}} f^{T} \leq f \mathbb{L}_{\widetilde{G}} f^{T} \leq(1+\epsilon) f \mathbb{L}_{\mathbb{G}} f^{T} .
$$

We note that edge-ranking can be accomplished using the quantities known as Green's values, which generalize the notion of effective resistance by allowing a damping constant. An edge-ranking algorithm for graphs using Green's values was studied extensively in [Chung and Zhao 10]. Here we will define a generalization of Green's values for connection graphs.

For $i=0, \ldots, n d-1$, let $\widehat{\phi}_{i}$ be the $i$ th eigenfunction of the normalized connection Laplacian $\mathbb{D}^{-1 / 2} \mathbb{L} \mathbb{D}^{-1 / 2}$ corresponding to eigenvalue $\lambda_{i}$. Define

$$
\mathbb{G}_{\beta}=\sum_{i=0}^{n d-1} \frac{1}{\lambda_{i}+\beta} \widehat{\phi}_{i}^{T} \widehat{\phi}_{i} .
$$

We remark that $\mathbb{G}_{\beta}$ can be viewed as a generalization of the pseudoinverse of the normalized connection Laplacian. Define the PageRank vector with a jumping constant $\alpha$ as the solution to the equation

$$
\widehat{\operatorname{pr}}_{\beta, \widehat{s}}=\frac{\beta}{2+\beta} \widehat{s}+\frac{2}{2+\beta} \widehat{\operatorname{pr}}_{\beta, \widehat{s}} \mathbb{Z}
$$


with $\beta=2 \alpha /(1-\alpha)$. These PageRank vectors are related to the matrix $\mathbb{G}_{\beta}$ via the following formula, which is straightforward to check:

$$
\frac{\widehat{p r}_{\beta, \widehat{s}}}{\beta}=s \mathbb{D}^{-1 / 2} \mathbb{G}_{\beta} \mathbb{D}^{1 / 2}
$$

Now for each edge $e=\{u, v\} \in E$, we define the connection Green's value $\widehat{g}_{\beta}(u, v)$ of $e$ to be the following combination of PageRank vectors:

$$
\begin{aligned}
\widehat{g}_{\beta}(u, v) & =\beta\left(\chi_{u}-\chi_{v}\right) \mathbb{D}^{-1 / 2} \mathbb{G}_{\beta} \mathbb{D}^{-1 / 2}\left(\chi_{u}-\chi_{v}\right)^{T} \\
& =\frac{\widehat{\operatorname{pr}}_{\beta, \chi_{u}}(u)}{d_{u}}-\frac{\widehat{p r}_{\beta, \chi_{u}}(v)}{d_{v}}+\frac{\widehat{\operatorname{pr}}_{\beta, \chi_{v}}(v)}{d_{v}}-\frac{\widehat{p r}_{\beta, \chi_{v}}(u)}{d_{u}} .
\end{aligned}
$$

This gives an alternative to the effective resistance as a technique for ranking edges. It could be used in place of the effective resistance in the edgesparsification algorithm.

\section{Eliminating Noise in Data Sets by Deleting Edges of High Rank}

In forming a connection graph, the possibility arises of there being erroneous data or errors in measurements, or other forms of "noise." This may be manifested in a resulting connection graph that is not consistent when it is expected that it would be. It is therefore desirable to be able to identify edges whose rotations are causing the connection graph to be inconsistent. We propose that a possible solution to this problem is to randomly delete edges of high rank in the sense of the edge-ranking. In this section, we will obtain bounds on the eigenvalues of the connection Laplacian resulting from the deletion of edges of high rank. This will have the effect of reducing the smallest eigenvalue, thus making the connection graph "closer" to being consistent, as seen in Theorem 2.2 .

To begin, we will derive a result on the spectrum of the connection Laplacian analogous to the result in [Chung and Radcliffe 11] on the adjacency matrix of a random graph.

Theorem 6.I. Let $\mathbb{G}$ be a given fixed connection graph with Laplacian $\mathbb{L}$. Delete edges $i j \in E(\mathbb{G})$ with probability $p_{i j}$. Let $\widehat{\mathbb{G}}$ be the resulting connection graph, $\widehat{\mathbb{L}}$ its connection Laplacian, and $\overline{\mathbb{Q}}=E(\widehat{\mathbb{L}})$. Then for $\epsilon \in(0,1)$, with probability at least $1-\epsilon$,

$$
\left|\lambda_{i}(\widehat{\mathbb{L}})-\lambda_{i}(\overline{\mathbb{L}})\right| \leq \sqrt{6 \Delta \ln (2 n d / \epsilon)},
$$

where $\Delta$ is the maximum degree, assuming $\Delta \geq \frac{2}{3} \ln (2 n d / \epsilon)$. 
To prove this, we need the concentration inequality from [Chung and Radcliffe 11].

Lemma 6.2. Let $X_{1}, \ldots, X_{m}$ be independent random $n \times n$ Hermitian matrices. Moreover, assume that $\left\|X_{i}-E\left(X_{i}\right)\right\|_{2} \leq M$ for all $i$, and put $v^{2}=$ $\left\|\sum \operatorname{Var}\left(X_{i}\right)\right\|_{2}$. Let $X=\sum X_{i}$. Then for all $a>0$,

$$
\operatorname{Pr}\left(\|X-E(X)\|_{2}>a\right) \leq 2 n \exp \left(-\frac{a^{2}}{2 v^{2}+2 M a / 3}\right) .
$$

Proof of Theorem 6.I. Our proof follows ideas from [Chung and Radcliffe 11]. For $i j \in E(\mathbb{G})$, define $\mathbb{A}^{i j}$ to be the matrix with rotation $O_{i j}$ in the $i, j$ position, $O_{j i}=O_{i j}^{T}$ in the $j, i$ position, and 0 elsewere. Define random variables $h_{i j}=1$ if the edge $i j$ is deleted, and 0 otherwise. Let $\mathbb{A}^{i i}$ be the diagonal matrix with $I_{d \times d}$ in the $i$ th diagonal position and 0 elsewhere. Then note that

$$
\widehat{\mathbb{L}}=\mathbb{L}+\sum_{i, j \in E} h_{i j} \mathbb{A}^{i j}-\sum_{i=1}^{n} \sum_{j \sim i} h_{i j} \mathbb{A}^{i i}
$$

and

$$
\overline{\mathbb{L}}=\mathbb{L}+\sum_{i, j \in E} p_{i j} \mathbb{A}^{i j}-\sum_{i=1}^{n} \sum_{j \sim i} p_{i j} \mathbb{A}^{i i}
$$

Therefore,

$$
\widehat{\mathbb{L}}-\overline{\mathbb{L}}=\sum_{i, j \in E}\left(h_{i j}-p_{i j}\right) \mathbb{A}^{i j}-\sum_{i=1}^{n} \sum_{j \sim i}\left(h_{i j}-p_{i j}\right) \mathbb{A}^{i i} .
$$

To use Lemma 6.2, we must compute the variances. We have

$$
\begin{aligned}
\operatorname{Var} & \left(\left(h_{i j}-p_{i j}\right) \mathbb{A}^{i j}\right) \\
\quad & =E\left(\left(h_{i j}-p_{i j}\right)^{2}\left(\mathbb{A}^{i j}\right)^{2}\right)=\operatorname{Var}\left(h_{i j}-p_{i j}\right)\left(\mathbb{A}^{i i}+\mathbb{A}^{j j}\right) \\
& =p_{i j}\left(1-p_{i j}\right)\left(\mathbb{A}^{i i}+\mathbb{A}^{j j}\right),
\end{aligned}
$$

and in a similar manner,

$$
\operatorname{Var}\left(\left(h_{i j}-p_{i j}\right) \mathbb{A}^{i i}\right)=p_{i j}\left(1-p_{i j}\right) \mathbb{A}^{i i}
$$


Algorithm 3. $\left(\mathbb{H}=\left(V, E^{\prime}, O, w^{\prime}\right)\right)=\operatorname{ReduceNoise}\left(\mathbb{G}=(V, E, O, w), p^{\prime}, q, \alpha\right)$.

1. Select $q$ edges in $q$ rounds. In each round, one edge is selected. Each edge $e$ is chosen with probability $p_{e}$ proportional to its effective resistance. Then the chosen edge is assigned a weight $w_{e}^{\prime}=w_{e} /\left(q p_{e}\right)$.

2. Delete $\alpha q=q^{\prime}$ edges in $q^{\prime}$ rounds. In each round, one edge is deleted. Each edge $e$ is chosen with probability $p_{e}^{\prime}$ proportional to the weight $w_{e}^{\prime}$.

3. Return $\mathbb{H}$, the connection graph resulting after the edges are deleted.

Therefore,

$$
\begin{aligned}
v^{2} & =\left\|\sum_{i, j \in E} p_{i j}\left(1-p_{i j}\right)\left(\mathbb{A}^{i i}+\mathbb{A}^{j j}\right)+\sum_{i=1}^{n} \sum_{i \sim j} p_{i j}\left(1-p_{i j}\right) \mathbb{A}^{i i}\right\|_{2} \\
& \leq 2\left\|\sum_{i=1}^{n}\left(\sum_{j=1}^{n} p_{i j}\left(1-p_{i j}\right)\right) \mathbb{A}^{i i}\right\|_{2}=2 \max _{i} \sum_{j=1}^{n} p_{i j}\left(1-p_{i j}\right) \\
& \leq 2 \max _{i} \sum_{j=1}^{n} p_{i j} \leq 2 \Delta .
\end{aligned}
$$

Each $\mathbb{A}^{i j}$ clearly has norm 1 , so we can take $M=1$. Therefore, by Lemma 6.2 , taking $a=\sqrt{6 \Delta \ln (2 n d / \epsilon)}$, we see that

$$
\begin{aligned}
\operatorname{Pr}\left(\|\widehat{\mathbb{L}}-\overline{\mathbb{L}}\|_{2}>a\right) & \leq 2 n d \exp \left(-\frac{a^{2}}{2 v^{2}+2 M a / 3}\right) \leq 2 n d \exp \left(-\frac{6 \Delta \ln (2 n d / \epsilon)}{6 \Delta}\right) \\
& =\epsilon .
\end{aligned}
$$

By a consequence of Weyl's theorem (see, for example, [Horn and Johnson 85]), since $\widehat{\mathbb{L}}$ and $\overline{\mathbb{L}}$ are Hermitian, we have

$$
\left|\lambda_{i}(\widehat{\mathbb{L}})-\lambda_{i}(\overline{\mathbb{L}})\right| \leq\|\widehat{\mathbb{L}}-\overline{\mathbb{L}}\|_{2} .
$$

The result then follows.

We now present our Algorithm 3 to delete edges of a connection graph with the goal of decreasing the smallest eigenvalue of the connection Laplacian.

Our analysis of this algorithm will combine Theorems 5.1 and 6.1. Given a connection graph $\mathbb{G}$, define $\lambda_{\mathbb{G}}$ to be the smallest eigenvalue of its connection Laplacian. 
Theorem 6.3. Let $\xi, \epsilon, \delta \in(0,1)$ be given. Given a connection graph $\mathbb{G}$ with $m$ edges,

$$
m>q=\frac{4 n d(\log (n d)+\log (1 / \xi))}{\epsilon^{2}}
$$

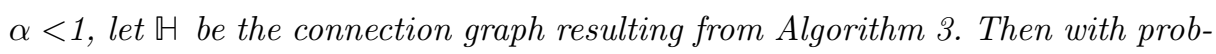
ability at least $(1-\xi)(1-\delta)$, the subgraph $\amalg$ satisfies

$$
\lambda_{H} \leq(1-\alpha+\epsilon) \lambda_{\mathbb{G}}+\sqrt{6 \Delta \ln (2 n d / \delta)},
$$

provided that the maximum degree $\Delta$ satisfies $\Delta \geq \frac{2}{3} \ln (2 n d / \delta)$.

Proof. We first note that with $\xi, \epsilon$, and $q$ as specified, the edge-selection procedure described in step 1 of Algorithm 3 is the same procedure described in Algorithm 2 and in Theorem 5.1. Let $\widetilde{\mathbb{G}}$ be the weighted graph resulting from the edge selection, and let $\mathbb{L}_{\widetilde{\mathbb{G}}}$ be its connection Laplacian. Then by Theorem 5.1, we know that with probability at least $\xi$, for every $f: V \rightarrow \mathbb{R}^{d}$, we have

$$
(1-\epsilon) f \mathbb{L}_{\mathbb{G}} f^{T} \leq f \mathbb{L}_{\widetilde{\mathbb{G}}} f^{T} \leq(1+\epsilon) f \mathbb{L}_{\mathbb{G}} f^{T} .
$$

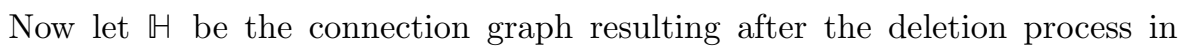
step 2 of Algorithm 3, and let $\mathbb{L}_{\mathbb{H}}$ be its connection Laplacian. We note that $\mathrm{H}$ is a random connection graph resulting from the deletion of edges of a fixed connection graph, as described in Theorem 6.1. Let $\overline{\mathbb{L}}_{\mathbb{H}}$ be the matrix of expected values of the entries of $\mathbb{L}_{H}, \overline{\mathbb{L}}_{\mathbb{H}}=E\left(\mathbb{L}_{H}\right)$. Note that the deletion procedure deletes $\alpha q$ of the $q$ edges from $\widetilde{G}$ with probability proportional to the weight on each edge, so that the expected value is $\overline{\mathbb{L}}_{\mathbb{H}}=\mathbb{L}_{\mathbb{G}}-\alpha \mathbb{L}_{\widetilde{\mathbb{G}}}$. From (6.1), it follows that

$$
f \mathbb{L}_{\mathbb{G}} f^{T}-(1+\epsilon) \alpha f \mathbb{L}_{\mathbb{G}} f^{T} \leq f\left(\mathbb{L}_{\mathbb{G}}-\alpha \mathbb{L}_{\widetilde{G}}\right) f^{T} \leq f \mathbb{L}_{\mathbb{G}} f^{T}-(1-\epsilon) \alpha f \mathbb{L}_{\mathbb{G}} f^{T},
$$

and thus

$$
f \mathbb{L}_{\mathbb{G}} f^{T}-(1+\epsilon) \alpha f \mathbb{L}_{\mathbb{G}} f^{T} \leq f \overline{\mathbb{L}}_{\mathbb{H}} f^{T} \leq f \mathbb{L}_{\mathbb{G}} f^{T}-(1-\epsilon) \alpha f \mathbb{L}_{\mathbb{G}} f^{T} .
$$

In particular, it follows that

$$
\frac{f \overline{\mathbb{\complement}}_{\mathbb{H}} f^{T}}{f f^{T}} \leq(1-\alpha+\epsilon) \frac{f \mathbb{\complement}_{\mathbb{G}} f^{T}}{f f^{T}}
$$

for every $f: V \rightarrow \mathbb{R}^{d}$, and therefore that

$$
\lambda_{0}\left(\overline{\mathbb{L}}_{\mathbb{H}}\right) \leq(1-\alpha+\epsilon) \lambda_{0}\left(\mathbb{L}_{\mathbb{G}}\right) .
$$

Finally, by Theorem 6.1 , given $\delta>0$, we have with probability at least $\xi(1-\delta)$ that

$$
\lambda_{H}<(1-\alpha+\epsilon) \lambda_{\mathbb{G}}+\sqrt{6 \Delta \ln (2 n d / \delta)} .
$$




\section{References}

[Achlioptas 01] D. Achlioptas. "Database-Friendly Random Projections." In Proceedings of the 20th ACM Symposium on Principles of Database Systems, pp. 274-281, 2001.

[Agarwal et al. 09] S. Agarwal, N. Snavely, I. Simon, S. M. Seitz, and R. Szeliski. "Building Rome in a Day." In Proceedings of the 12th IEEE International Conference on Computer Vision, pp. 72-79, 2009.

[Andersen et al. 06] R. Andersen, F. Chung, and K. Lang. "Local Graph Partitioning Using PageRank Vectors." Proceedings of the 47th IEEE Symposium on Foundations of Computer Science, pp. 475-486, 2006.

[Anderson et al. 10] G. W. Anderson, A. Guionnet, and O. Zeitouni. An Introduction to Random Matrices. Cambridge University Press, 2010.

[Bandeira et al. 12] A. S. Bandeira, A. Singer, and D. A. Spielman. "A Cheeger Inequality for the Graph Connection Laplacian." Available online (http://arxiv.org/ pdf/1204.3873v1.pdf, 2012.

[Benczúr and Karger 96] A. A. Benczúr and D. R. Karger. "Approximating $s-t$ Minimum Cuts in $\tilde{O}\left(n^{2}\right)$ Time." In Proceedings of the 28th ACM Symposium on Theory of Computing, pp. 47-55, 1996.

[Berkhin 06] P. Berkhin. "Bookmark-Coloring Approach to Personalized PageRank Computing." Internet Mathematics 3 (2006), 41-62.

[Borgs et al. 12] C. Borgs, M. Brautbar, J. Chayes, and S.-H. Teng. "A Sublinear Time Algorithm for PageRank Computations." In Proceedings of the 9th International Workshop on Algorithms and Models for the Web Graph, pp. 49-53, 2012.

[Brin and Page 98] S. Brin and L. Page. "The Anatomy of a Large-Scale Hypertextual Web Search Engine." Computer Networks and ISDN Systems 30 (1998), 107-117.

[Chung and Radcliffe 11] F. Chung and M. Radcliffe. "On the Spectra of General Random Graphs." Electronic Journal of Combinatorics 18 (2011), 215-229.

[Chung and Sternberg 92] F. Chung and S. Sternberg. "Laplacian and Vibrational Spectra for Homogeneous Graphs." J. Graph Theory 16 (1992), 605-627.

[Chung and Zhao 10] F. Chung and W. Zhao. "A Sharp PageRank Algorithm with Applications to Edge Ranking and Graph Sparsification." In Proceedings of Workshop on Algorithms and Models for the Web Graph (WAW) 2010, pp. 2-14. Stanford, California, 2010.

[Cristofides and Markström 08] D. Cristofides and K. Markström. "Expansion Properties of Random Cayley Graphs and Vertex Transitive Graphs via Matrix Martingales." Random Structures Algorithms 32 (2008), 88-100.

[Cucuringu et al. 12] M. Cucuringu, Y. Lipman, and A. Singer. "Sensor Network Localization by Eigenvector Synchronization over the Euclidean Group." ACM Transactions on Sensor Networks 8 (2012), No. 19.

[Firat et al. 07] A. Firat, S. Chatterjee, and M. Yilmaz. "Genetic Clustering of Social Networks Using Random Walks." Computational Statistics and Data Analysis 51 (2007), 6285-6294. 
[Fouss et al. 07] F. Fouss, A. Pirotte, J.-M. Renders, and M. Saerens. "Random-Walk Computation of Similarities between Nodes of a Graph with Application to Collaborative Recommendation." Knowledge and Data Engineering 19 (2007), 355-369.

[Golub and Van Loan 96] G. H. Golub and C. F. Van Loan. Matrix Computations, 3rd ed. Johns Hopkins Univ. Press, 1996.

[Hadani and Singer 11] R. Hadani and A. Singer. "Representation Theoretic Patterns in Three Dimensional Cryo-electron Microscopy I: The Intrinsic Reconstitution Algorithm." Annals of Mathematics 174 (2011), 1219-1241.

[Herbster et al. 08] M. Herbster, M. Pontil, and Sergio Rojas. "Fast Prediction on a Tree." Proceedings of the Neural Information Processing Systems Foundation 21 (2008), 657-664.

[Horn and Johnson 85] R. Horn and C. Johnson. Matrix Analysis. Cambridge University Press, 1985.

[Jeh and Widom 03] G. Jeh and J. Widom. "Scaling Personalized Web Search." Proceedings of the 12th World Wide Web Conference WWW, pp. 271-279, 2003.

[Jolliffe 02] I. T. Jolliffe. Principal Component Analysis, Springer Series in Statistics, 2nd ed. Springer, 2002.

[Karger 94] D. R. Karger. "Using Randomized Sparsification to Approximate Minimum Cuts." In Proceedings of the 15th ACM symposium on Discrete Algorithms, pp. 424432, 1994.

[Karger 99] D. R. Karger. "Random Sampling in Cut, Flow, and Network Design Problems." Mathematics of Operations Research 24, (1999), 383-413.

[Karger 00] D. R. Karger, "Minimum Cuts in Near-Linear Time." Journal of the ACM 47 (2000), 46-76.

[Kirchhoff 47] F. Kirchhoff. "Über die Auflösung der Gleichungen, auf welche man bei der Untersuchung der linearen Verteilung galvanischer Ströme geführt wird." Ann. Phys. chem. 72 (1847), 497-508.

[Koutis 10] I. Koutis, G. L. Miller, and R. Peng. "Approaching Optimality for Solving SDD Linear Systems." In Proceedings of 51st IEEE Symposium on Foundations of Computer Science, pp. 235-244, 2010.

[Lowe 99] D. G. Lowe. "Object Recognition from Local Scale-Invariant Features." In Proceedings of the 7th IEEE International Conference on Computer Vision, pp. 11501157, 1999.

[Penrose 55] R. Penrose. "A Generalized Inverse for Matrices." Cambridge Philosophical Society 51 (1955), 406-413.

[Recht 11] B. Recht. "Simpler Approach to Matrix Completion." Journal of Machine Learning Research 12 (2011), 3413-3430.

[Singer 11] A. Singer. "Angular Synchronization by Eigenvectors and Semidefinite Programming." Applied and Computational Harmonic Analysis 30, (2011), 20-36.

[Singer and Wu 12] A. Singer and H.-T. Wu. "Vector Diffusion Maps and the Connection Laplacian." Communications on Pure and Applied Mathematics 65 (2012), $1067-1144$. 
[Singer et al. 11] A. Singer, Z. Zhao Y. Shkolnisky, and R. Hadani. "Viewing Angle Classification of Cryo-electron Microscopy Images Using Eigenvectors." SIAM Journal on Imaging Sciences 4 (2011), 723-759.

[Spielman and Srivastava 08] D. A. Spielman and N. Srivastava. "Graph Sparsification by Effective Resistances." In Proceedings of 40th ACM symposium on Theory of Computing, pp. 563-568, 2008.

[Spielman and Teng 04] D. A. Spielman and S.-H. Teng. "Nearly-Linear Time Algorithms for Graph Partitioning, Graph Sparsification, and Solving Linear Systems." In Proceedings of the 36th ACM Symposium on Theory of Computing, pp. 81-90, 2004.

[Spielman and Teng 06] D. A. Spielman and S.-H. Teng. "Nearly-Linear Time Algorithms for Preconditioning and Solving Symmetric, Diagonally Dominant Linear Systems." Available online (http://www.arxiv.org/abs/cs.NA/0607105), 2006.

[Spielman and Teng 10] D. A. Spielman and S.-H. Teng, "Spectral Sparsification of Graphs." SIAM Journal on Computing 40 (2011), 981-1025.

[Tropp 11] J. Tropp. "User-Friendly Tail Bounds for Sums of Random Matrices." Available online (http://arxiv.org/abs/1004.4389), 2011.

[Vershynin 08] Roman Vershynin. "A Note on Sums of Independent Random Matrices after Ahlswede-Winter." Available online (http://www-personal.umich.edu/ $\sim$ romanv/teaching/reading-group/ahlswede-winter.pdf), 2008.

[Vu 07] V. Vu. "Spectral Norm of Random Matrices." Combinatorica 27 (2007), 721736.

[Wigderson and Xiao 08] A. Wigderson and D. Xiao. "Derandomizing the AhlswedeWinter Matrix-Valued Chernoff Bound Using Pessimistic Estimators, and Applications." Theory of Computing 4 (2008), 53-76.

Fan Chung, Department of Department of Computer Science and Engineering, University of California, San Diego, 9500 Gilman Drive, La Jolla, CA 92093, USA (fan@ucsd.edu)

Wenbo Zhao, Department of Department of Computer Science and Engineering, University of California, San Diego, 9500 Gilman Drive, La Jolla, CA 92093, USA (w3zhao@ucsd.edu)

Mark Kempton, Department of Department of Computer Science and Engineering, University of California, San Diego, 9500 Gilman Drive, La Jolla, CA 92093, USA (mkempton@ucsd.edu) 